\section{Zur Chemie von Cannabis}

Turner et al. beschrieben 1980 in ihrer Übersicht insgesamt 421 Bestandteile der Hanfpflanze. Seither wurden einige weitere Inhaltsstoffe entdeckt, so daß heute von 430-450 Verbindungen ausgegangen werden kann, die im Hanf - allerdings nicht alle in einer einzigen Pflanze - gefunden werden können. Die meisten dieser Inhaltsstoffe finden sich ubiquitär in anderen pflanzlichen und tierischen Organismen, darunter Terpene (etwa 100), Hydrokarbone (50), Zucker und verwandte Verbindungen (35), einfache Säuren (20), Aminosäuren (18), Proteine, quaternäre Basen, ein Amid, Amine, Spermidinalkaloide, Alkohole, Aldehyde, Ketone, einfache Ester und Lactone, Steroide, nichtcannabinoide Phenole, Flavonoidglykoside, Vitamine sowie 2 Pigmente.

Die spezifischen Inhaltsstoffe sind die etwa 60 Cannabinoide, die sich mehrheitlich 10 Typen zuordnen lassen, von denen vor allem $\operatorname{der} \Delta^{9}$-Tetrahydrocannabinol-Typ $\left(\Delta^{9}\right.$-THC) mit 9 Vertretern, daneben der Cannabidiol-Typ (CBD), der Cannabigerol-Typ (CBG) und der Cannabichromen-Typ (CBC) die größte pharmakologische Bedeutung besitzen. Letztere 3 wirken nicht psychotrop. Vor allem dem Cannabidiol wird eine Modulation der THC-Wirkung bei der Verwendung von Ganzpflanzenzubereitungen zugeschrieben, was die gelegentlich beschriebene bessere Verträglichkeit gegenüber der Monosubstanz erklären kann, da es die psychotropen Wirkungen des THC antagonisiert. Cannabinoide wurden bisher nur in der Hanfpflanze entdeckt. In einer Pflanze finden sich meistens nur 3-4 Cannabinoide in relevanter Konzentration. Leitcannabinoid des zur Rauschmittelgewinnung genutzten Drogenhanfes ist das (-)- $\Delta^{9}$-trans-Tetrahydrocannabinol (Dronabinol), Leitcannabinoid des zur Faser- und Ölgewinnung verwendeten Faserhanfes ist Cannabidiol. Pharmakologisch wirksam sind die phenolischen Formen der Cannabinoide, die durch kurze Erhitzung aus den in der Pflanze vorwiegend - zu mehr als $95 \%$ - vorliegenden carboxylierten Formen entstehen.

\section{Literatur}

Turner CE, Elsohly MA, Boeren EG: Constituents of Cannabis sativa L. XVII. A review of the natural constituents. J Nat Prod 1980;43:169-234.

\section{KARGER}

Fax +497614520714

E-mailkargergmbh@aol.com

www.karger.com (c) 1999 S. Karger GmbH, Freiburg 